\section{Shaking the tree of life}

The Nature of Diversity: An Evolutionary Voyage of Discovery

by Daniel R. Brooks \& Deborah A.

McLennan

University of Chicago Press: 2002. 680 pp.

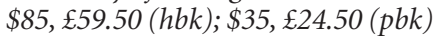

\section{Sean Nee}

"The Nature of Diversity is both phylogenetics and the environment, inextricably interwoven, rich in anachronisms and serendipity." Anachronisms and serendipity? This weird summary of the book is on the back cover. In fact, by virtue of its weirdness, perhaps it is an excellent description of a book with content such as this: "Darwinism is summarized by the Jagger principle: You can't always get what you want, but if you try sometime, you just might find you get what you need." This aperçu is actually highlighted for our special attention.

Biological diversity can be described by phylogenies - family trees that show the relationships between species. Such fundamental evolutionary information can be used for many purposes, such as identifying biological or environmental variables that promote speciation. The Nature of Diversity aims to tell us how to construct phylogenies, and then the sorts of things that we can then do with them. Brooks and McLennan give us an idiosyncratic view of these subjects, to say the least.

To construct a phylogeny, you need data pertaining to the species' relationships and a technique to extract the phylogenetic information from that data. Readers of Nature will have encountered many papers that present phylogenetic trees constructed by maximum-likelihood methods and, more recently, by bayesian methods, applied to molecular sequence data. To get a good phylogeny quickly, all you need to do is feed the output from your automated sequencer into a standard phylogenetic computer package and presto! - you have a phylogeny constructed using some of the most powerful and advanced techniques, theoretical and empirical, of the past 20 years.

But if you wish to learn something about these modern techniques you must look elsewhere. All you will learn from this book about maximum-likelihood methods is that the authors do not like them much, whereas bayesian methods do not seem to exist at all. I did not carefully study the authors' preferred recipe for phylogeny construction; suffice it to say that it has such ingredients as "Hennig's auxiliary principle" and "Remane's criteria". Nature readers will rarely encounter such arcana.

The authors are not crazy either about molecular sequence data. This is unfortu- nate, as molecules nullify some apparently nity, judging by the space given to their discussion. For example, what is a species? This question has different meanings in different contexts. Here's one. Consider a simple phylogeny consisting of, say, just humans, chimpanzees and the lineage that gave rise to us both. Certainly, humans and chimpanzees are different species. But what about the ancestral lineage? Is it a distinct, third species? It seems that there are different schools of thought on this question of questionable importance, differing in how they react to a situation in which this ancestor has no 'characters' that are different to those of, say, chimps. Well, even though we do not have the sequence data, we can know that the lineage had differences in molecular characters, so we need trouble ourselves no further and can turn to more interesting questions.

This book does become useful in the chapters that summarize numerous studies that have used phylogenies to make inferences in many different areas of enquiry, such as biogeography, coevolution and adaptive evolution. I learned of many interesting studies that I would probably not otherwise have encountered. But problems frequently arise in the synthesis of the material. Consider, for example, questions about 'radiations'. Say you have two taxonomic families of the same age, but one has twice as many species as the other. Is that remarkable, or could it reasonably have arisen by chance alone, even if they had the same speciation probabilities over time? Another question: I have numerous pairs of families, and in each of these pairs, one family has only blue species and the other only green grand concerns of the phylogenetic commu-

species and, in each pair, the blue family has more species than the green family. Is this remarkable, or might the apparent association between colour and species richness have readily arisen by chance alone? Alas, these two questions are inextricably conflated in this book, although they are actually completely different and must be kept well separated for correct analysis.

This big book has about 3,000 references, but this abundance is generated largely as a result of long lists of randomly important references to a topic, rather than just a few references to definitive works. So, for example, on ecological diversity indices we are not referred to Anne Magurran's excellent monograph Ecological Diversity and its Measurement (Princeton University Press, 1992). Instead we get a seemingly arbitrary list including such unicorns as a French paper from 1908 published in "Bull. Soc. Vaud. Sci. Nat." And what are we supposed to do with a list of 45 references to phylogenetically based species concepts? It is as if a Java Web-bot has been set loose to gorge itself on the web of science with a few key words for guidance and has then emptied itself in the privacy of the book's back pages in one giant, ghastly, purgative dump.

Many years ago there was a Punch cartoon in which a bishop apologizes to his timid curate for giving him a bad egg at breakfast. Not wanting to give offence, the curate replies: "Oh, no, my Lord, I assure you that parts of it are excellent!" I find myself in the position of the curate: this book is much like the curate's egg, in the original sense of the phrase.

Sean Nee is in the Division of Biological Sciences,

University of Edinburgh, Edinburgh EH93JT, UK.

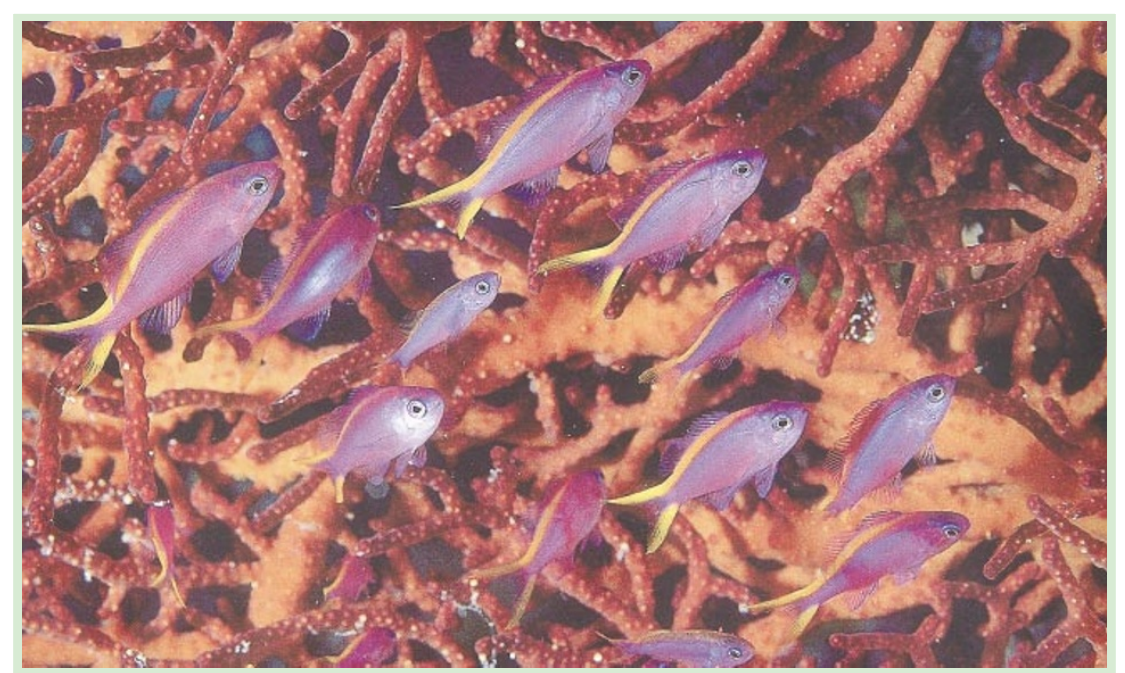

\section{A splash of colour}

The Great Barrier Reef is a living expanse of coral that stretches for over 2,000 kilometres along the east coast of Australia. It is home to an enormous variety of colourful fish, such as the purple anthias shown above. A collection of stunning photographs of the reef by David Doubilet is now published in Great Barrier Reef (National Geographic Society, \$40). 